\title{
DOCUMENTACIÓN A PRESENTAR Y EXEQUÁTUR DE SENTENCIA EXTRANJERA DE DIVORCIO: COMENTARIO AL AUTO DE LA AUDIENCIA PROVINCIAL DE BARCELONA, DE 20 DE NOVIEMBRE DE 2019
}

\author{
REQUIRED DOCUMENTS AND RECOGNITION AND \\ ENFORCEMENT OF FOREIGN DIVORCE JUDGEMENT: \\ COMMENTARY TO THE JUDICIAL DECREE OF THE \\ PROVINCIAL COURT OF BARCELONA, OF NOVEMBER 20, 2019
}

\author{
ENRIQUe FERNÁNDEZ MASIÁ \\ Profesor Titular de Derecho Internacional Privado \\ Universidad de Castilla-La Mancha \\ ORCID ID: 0000-0002-2062-967X
}

Recibido: 20.01.2020 / Aceptado: 27.03.2020

DOI: https://doi.org/10.20318/cdt.2020.5647

Resumen: El presente comentario analiza el auto dictado por la Audiencia Provincial de Barcelona en relación con los documentos que son necesarios presentar junto con la demanda de exequátur de una sentencia argentina de divorcio, para que sea admitida a trámite de acuerdo con lo previsto en la Ley de Cooperación Jurídica Internacional en Materia Civil.

Palabras clave: reconocimiento, exequátur, documentos necesarios, cooperación judicial internacional en materia civil.

Abstract: This paper analyzes the Judicial Decree of the Provincial Court of Barcelona about the required documents that have to file along the claim of recognition and enforcement of a divorce judgment obtained in Argentina, as provided for the Spanish Act on International Judicial Cooperation in Civil Matters.

Keywords: recognition, exequatur proceedings, required documents- international judicial, cooperation in civil matters.

Sumario: I Introducción. II. Los hechos que dan lugar al recurso ante la Audiencia Provincial de Barcelona. III. Estimación del recurso de apelación y valoración de la admisión a trámite de la demanda de exequátur de la sentencia argentina de divorcio.

\section{Introducción}

1. El objeto del presente comentario es el Auto dictado por la Audiencia Provincial de Barcelona, Sección Dieciocho, el 20 de noviembre de 2019 (en adelante, AAP de Barcelona) ${ }^{1}$, por el cual se estima el recurso de apelación interpuesto contra el Auto dictado por el Juzgado de Primera Instancia e Instrucción

${ }^{1}$ ECLI:ES:APB:2019:9452A. 
número 6 de Sant Boi de Llobregat, de 13 de mayo de 2019 (en adelante AJPI de Sant Boi de Llobregat), mediante el cual se había acordado la inadmisión de la demanda de exequátur de una sentencia de divorcio pronunciada por los tribunales argentinos, por no cumplirse los requisitos documentales previstos por la Ley de Cooperación Judicial Internacional en Materia Civil (en adelante LCJIMC) ${ }^{2}$. Como puede fácilmente comprobarse se trata de un tema de especial relevancia en la práctica pues es, sin duda, uno de los que mayores quebraderos de cabeza supone para los abogados cuando en nombre de su cliente y representado, buscan dotar de eficacia a las resoluciones extranjeras de divorcio en nuestro país.

2. El sector del reconocimiento y ejecución de las decisiones extranjeras es un sector del Derecho Internacional Privado (en adelante DIPr), que en los últimos años está sufriendo enormes cambios y novedades en España. La modernización de nuestro sistema no sólo ha venido dada por la frenética actividad de las instituciones europeas a la hora de desarrollar sus competencias en el ámbito del DIPr, sino también por la esperada modernización, después de mucho tiempo, del régimen estatal de reconocimiento y ejecución mediante la adopción de la LCJIMC en 2015, que ha venido a sustituir a la antigua regulación contemplada en la Ley de Enjuiciamiento Civil de $1881^{3}$.

3. Hoy en día, las sentencias extranjeras de divorcio pueden obtener el reconocimiento en nuestro país a través de distintos instrumentos legales ${ }^{4}$. Por ello, una primera cuestión práctica de gran importancia consiste en identificar el régimen jurídico aplicable a una concreta sentencia extranjera en esta materia. Y ello porque dependiendo del mismo, existen diferencias muy relevantes en relación con los mecanismos posibles para hacer efectiva dicha resolución, los motivos de rechazo, la autoridad competente, el procedimiento a seguir y, por lo que atañe a la cuestión concreta de este comentario, la documentación a presentar.

4. Para la correcta delimitación del régimen jurídico aplicable hay que tomar en consideración la procedencia u origen de la concreta sentencia de divorcio ${ }^{5}$. Así, en primer lugar, y con primacía, hemos de tomar en consideración el régimen europeo. A las resoluciones dictadas por las autoridades públicas de los Estados miembros de la Unión Europea - excepto Dinamarca- que declaran el divorcio, es aplicable actualmente el Reglamento 2201/2003, de 27 de noviembre de 2003 (Reglamento Bruselas II-bis) Hay que tener en cuenta, sin embargo, que muy recientemente, el 2 de julio de 2019 se ha publicado en el Diario Oficial de la Unión Europa (número L 178), un nuevo texto legislativo, que vendrá a sustituir a aquel. Es el Reglamento 2019/1111 relativo a la competencia, el reconocimiento y la ejecución de resoluciones en materia matrimonial y de responsabilidad parental y sobre sustracción internacional de menores, que es conocido ya comúnmente como Reglamento Bruselas II ter o Bruselas II bis versión refundida (recast) y, que entrará en vigor, con carácter general, el 1 de agosto de $2022^{7}$.

5. Descartado este régimen jurídico como aplicable al procedimiento concreto en este caso, ya que la resolución ha sido emitida por parte de las autoridades judiciales de un tercer Estado, tendríamos que tomar en consideración la red de convenios internacionales bilaterales de los que España es Estado parte en esta materia. Y aquí hay que señalar, lógicamente, que tras la adopción del Reglamento Bruselas

\footnotetext{
${ }^{2}$ BOE, núm. 182, de 31 de julio de 2015.

${ }^{3}$ A este respecto, puede consultarse, A. Rodriguez Benot, "La Ley de Cooperación Jurídica Internacional en Materia Civil”, Cuadernos de Derecho Transnacional, $\mathrm{n}^{\circ} .1$, vol.8, 2016, pp.234-259; F. GASTÓN INCHAUSTI, "Reconocimiento y ejecución de resoluciones judiciales extranjeras en la Ley de Cooperación Jurídica Internacional en materia civil”, Cuadernos de Derecho Transnacional, $\mathrm{n}^{\mathrm{0}} .2$, Vol.7, 2015, pp.158-187.

${ }^{4}$ Vid. A.L. Calvo Caravaca y J. Carrascosa Gonzalez, Derecho internacional privado, Vol.II, 18 ed., Comares, Granada, 2018, pp.296-306.

${ }^{5}$ C. Esplugues Mota, J.L. Iglesias Buhigues y G. Palao Moreno, Derecho internacional privado, 13 ed., Valencia, Tirant lo Blanch, 2019, p.495.

${ }^{6}$ J.I. Paredes Perez, "El sistema europeo de reconocimiento y ejecución de decisiones en materia de Derecho de familia: crisis matrimoniales y pronunciamientos vinculados", La Ley Derecho de familia, no.17, enero-marzo, 2018, p.3.

7 Vid. A. Borras Rodriguez, "Bruselas II, Bruselas II bis, Bruselas ter...", Revista electrónica de estudios internacionales, $\mathrm{n}^{\circ} .38,2019$.
} 
2201/2003, los convenios bilaterales con Francia, Italia, Austria, Alemania, Checoslovaquia (para República Checa y Eslovaquia) y Bulgaria ya no serían aplicables. Sin embargo, quedan activos en materia de reconocimiento y ejecución de resoluciones judiciales sobre divorcio, los convenios bilaterales con terceros Estados que si incluyen esta cuestión dentro de su ámbito de aplicación material. A saber, los convenios con Suiza, Colombia, China, Rusia -Convenio con la antigua URSS-, Marruecos, Túnez, Argelia y Mauritania.

6. Dado que la sentencia objeto de la solicitud de reconocimiento ante nuestros tribunales ha sido dictada por parte de los tribunales argentinos, y no existe convenio bilateral aplicable en este supuesto, ha de recurrirse a la aplicación del régimen estatal contemplado en la LCJIMC (artículos 41 a 61). A pesar del carácter subsidiario de este régimen, sin embargo, se ha de subrayar que el mismo en la práctica adquiere una gran importancia, no sólo en relación con la cuestión que nos ocupa, pues se refiere a cualquier materia litigiosa civil y mercantil, a salvo de la legislación sectorial específica, sino porque cubre una muy extensa lista de posibles supuestos fácticos, “...habida cuenta que en los tratados y reglamentos de la Unión Europea rige el principio de reciprocidad, de forma que sólo son aplicables respecto a resoluciones judiciales originarias de Estados parte en dichos instrumentos y relativas a materias cubiertas por los mismos"8.

7. Identificado el régimen jurídico aplicable en este concreto caso, en las próximas páginas se analizará, en primer lugar, los elementos fácticos que dieron lugar tanto al AJPI de Sant Boi de Llobregat que no admitió a trámite la demanda de exequátur como al correspondiente recurso de apelación resuelto de forma favorable mediante el AAP de Barcelona. En segundo lugar, se valorarán los elementos jurídicos que han sido analizados en el auto objeto del presente comentario, especialmente los referidos a la documentación a presentar junto con la demanda de exequátur y que están previstos en la LCJIMC.

\section{Los hechos que dan lugar al recurso ante la Audiencia Provincial de Barcelona}

8. Los hechos que motivan la resolución objeto de comentario son los siguientes: el procedimiento se inició mediante la presentación ante el Juzgado de Primera Instancia de Sant Boi de Llobregat de una demanda de exequátur de una sentencia de divorcio pronunciada por el Juzgado de Familia número 4 del Departamento Judicial de Mar de Plata, Provincia de Buenos Aires de la República Argentina. En el fallo de esta resolución se decretaba el divorcio vincular de los cónyuges por la causal prevista por el artículo 214.2 del Código civil argentino que se refiere a la separación de hecho sin voluntad de unirse por un lapso temporal de más de tres años. En el AJPI de Sant Boi de Llobregat se inadmite esta demanda de exequátur, argumentándose que no consta la firmeza y fuerza ejecutiva de la resolución extranjera, ni se ha aportado el original o copia auténtica de esta sentencia legalizada o apostillada, de conformidad con lo previsto en el artículo 54 de la LCJIMC, por lo que finalmente se acuerda archivar el procedimiento.

9. Ante este Auto de primera instancia, se interpone recurso de apelación, cuyas alegaciones se fundamentan por la parte recurrente en que se ha aportado la resolución extranjera apostillada por conducto telemático y con expresión de firmeza, no habiendo sido requerida a presentarla de manera original. Por ello se argumenta que se ha vulnerado por el auto recurrido, el artículo 24.1 de la Constitución española en su vertiente de acceso a la jurisdicción, solicitándose que se reconozca y se declare la ejecutoriedad en España de la sentencia de divorcio.

\section{Estimación del recurso de apelación y valoración de la admisión a trámite de la demanda de exequátur de la sentencia argentina de divorcio}

10. En la resolución en donde se admite el recurso de apelación se comienza señalando cuales son los documentos que de acuerdo con lo previsto en la LCJIMC deben acompañar la demanda de

\footnotetext{
${ }^{8}$ Preámbulo de la LCJIMC.
} 
exequátur. En principio, se declara que son esencialmente los mismos que los requeridos por la antigua ley procesal de 1881, enumerándose: "1) original y copia auténtica, legalizada o apostillada de la resolución extranjera, 2) documento acreditativo de la firmeza, pudiendo constar la misma en la propia resolución, lo que supone una garantía legal a la hasta ahora facultad potestativa de los juzgados de solicitar en documento independiente dich a firmeza, so pena de no admitir a trámite el exequátur; y por último, 3) las traducciones, que atendiendo al artículo 144.2 LEC, podrán ser "privadas", es decir, no juradas, sin perjuicio de que la contraparte pueda impugnarlas por tenerlas por no fieles".

11. Hay que señalar que para conseguir el reconocimiento a título principal y con efectos erga omnes de la sentencia argentina de divorcio en España se requiere seguir los trámites del procedimiento de exequátur contemplado en el artículo 54 de la LCJIMC $^{9}$. En esta disposición en su apartado 4 se señala como este procedimiento ha de iniciarse mediante demanda, que debe ajustarse a lo previsto en el artículo 399 de la Ley de Enjuiciamiento Civil -en adelante LEC-, no siendo, por tanto, diferente en sus requisitos formales a la demanda que pudiera iniciar un proceso declarativo civil. Junto con ella, y aquí podemos comparar con lo dicho en la AAP de Barcelona para evaluar si se ha actuado de manera correcta, se requiere necesariamente la presentación de otros documentos. El juez español, dado el principio documental que rige este procedimiento de exequátur -en donde no es posible la celebración de vista oral ni la práctica de actos probatorios-, habrá de mostrar una actitud cautelosa y comprobar de manera fehaciente que toda la documentación requerida se ha presentado de manera correcta y se adecúa a lo previsto en nuestra legislación ${ }^{10}$.

12. De lo dicho con anterioridad, igualmente, se puede derivar el extremo cuidado con que el abogado de la parte solicitante habrá de presentar toda la documentación exigida y que ésta sea conforme a las exigencias formales previstas. Pues si bien es verdad que, en principio, después del examen por parte del letrado de la administración de justicia, debería concederse un plazo de subsanación de cinco días, la falta de los documentos exigidos o su inadecuada presentación tiene como consecuencia inmediata la inadmisión de la demanda de exequátur, con los consiguientes perjuicios para su cliente y representado. Hemos de señalar que, sin embargo, estos perjuicios no son irreparables, dado que la inadmisión a trámite no impide, sin embargo, que el solicitante, si se subsanan los defectos formales, pueda volver a instar el reconocimiento mediante la presentación de una nueva demanda de exequátur, por lo que, en consecuencia, dicha inadmisión no tiene los efectos de cosa juzgada, pero si alargaría en gran medida todo el procedimiento, con los consiguientes costes tanto en tiempo como en dinero para la parte interesada en el reconocimiento de la sentencia extranjera en nuestro país.

13. En concreto, El artículo 54.4 de la LCJIMC ${ }^{11}$ señala que la demanda deberá ir acompañada de: a) El original o copia auténtica de la resolución extranjera, debidamente legalizados o apostillados; b) El documento que acredite, si la resolución se dictó en rebeldía, la entrega o notificación de la cédula de emplazamiento o el documento equivalente; c) Cualquier otro documento acreditativo de la firmeza y fuerza ejecutiva en su caso de la resolución extranjera en el Estado de origen, pudiendo constar este extremo en la propia resolución o desprenderse así de la ley aplicada por el tribunal de origen; d) Las traducciones pertinentes con arreglo al artículo 144 de la Ley de Enjuiciamiento Civil.

13. En primer lugar, por tanto, y así lo señala de manera correcta el propio AAP de Barcelona, se requiere la presentación de la sentencia original o copia auténtica, dotada de su correspondiente legalización o "apostilla". Esta exigencia documental, que siempre ha sido exigida por nuestra normativa proce-

\footnotetext{
9 F.J. Garcimartin Alferez, Derecho internacional privado, 4 ed., Cizur Menor, Civitas, 2019, p.273.

10 A.L. Calvo Caravaca y J. Carrascosa Gonzalez, Derecho internacional privado, Vol.I, 18 ed., Granada, Comares, 2018, p.459.

11 Vid. en profundidad sobre esta disposición, P. MARTINEz Llorente, "Comentario al artículo 54" en Comentario a la Ley 29/2015 de Cooperación Jurídica Internacional en materia civil, A. Fernandez-TresGUERRES GARCIA (coord..), Barcelona, Bosch, 2017, pp.458-472; J. I. PARedes Perez, "Comentario al artículo 54" en Comentarios a la Ley de Cooperación Jurídica Internacional en Materia Civil, F.P. Mendez Gonzalez y G. Palao Moreno (Dirs.), Valencia, Tirant lo Blanch, 2017 , pp.628-663.
} 
sal, tiene como fin evitar todo tipo de falsedades o fraudes, esto es, que lo que verdaderamente se presenta sea una "auténtica" resolución extranjera. Una resolución que habrá de presentarse en su totalidad y conforme a las exigencias formales previstas por el Derecho del Estado de origen de la sentencia. Y, además, dicha sentencia deberá estar legalizada conforme a lo previsto en el 323 de la LEC o, en su caso, venir acompañada de su correspondiente apostilla, de acuerdo con lo previsto en el Convenio de la Haya de 5 de octubre de 1961, suprimiendo la exigencia de la legalización de los documentos públicos extranjeros ${ }^{12}$.

14. La falta de este primer documento había sido uno de los motivos para inadmitir a trámite la demanda por parte del AJPI de Sant Boi de Llobregat. Y acertadamente, el AAP de Barcelona rechaza de plano esta idea, tomando en consideración que este requisito había sido convenientemente cumplido por la parte solicitante, al remitir por vía telemática, copia de la sentencia apostillada. Más en concreto, como documento independiente aportado se incluye de manera completa la resolución y en todos los folios que la integran figura el siguiente texto "certifico que la presente es conforme con su original que en este acto tengo a la vista. Conste. Mar de Plata, 5 de noviembre de 2014, junto al sello e impresión del secretario". Y junto con la sentencia, además, como otro documento independiente se había aportado la apostilla correspondiente.

15. Como un segundo documento a aportar junto con la demanda, se precisa en el artículo 54.4 de la LCJIMC, que el demandante presente aquel que acredite, si la resolución se dictó en rebeldía, la entrega o notificación de emplazamiento o el documento equivalente que pruebe que el demandado fue citado en tiempo y forma, y podía haber comparecido. Esta exigencia documental como requisito ineludible para la admisión a trámite de la demanda supone la prueba de que el demandado en el pleito de origen tuvo oportunidad de defenderse ante los tribunales extranjeros y que, si no lo hizo, fue porque no quiso voluntariamente hacerlo ${ }^{13}$. En relación con la resolución comentada, el AAP de Barcelona no menciona entre la documentación a presentar, este concreto documento, ni tampoco lo había hecho con anterioridad el propio AJPI de Sant Boi de Llobregat, pero la razón ha de encontrarse en que, en este caso concreto, no se dio la circunstancia requerida para tener que aportarlo, ya que la demanda de divorcio fue planteada conjuntamente y de mutuo acuerdo por ambos cónyuges ante los tribunales argentinos.

16. Un tercer documento, en principio, que se requiere junto con la demanda, es aquel que acredite la firmeza y, en su caso, fuerza ejecutiva de la resolución extranjera en el Estado de origen ${ }^{14}$. Con la acreditación de la firmeza, el legislador español quiere evitar el reconocimiento de efectos a sentencias que puedan ser esencialmente revocables ${ }^{15}$. La falta de constancia de la firmeza y de "su fuerza ejecutiva" de la resolución extranjera fue el segundo de los motivos por los que se inadmitió la demanda de exequátur por parte del AJPI de Sant Boi de Llobregat. Frente a ello, el AAP de Barcelona señala correctamente como el tenor literal del artículo 54.4 de la LCJIMC, ya no obliga a presentar un documento independiente que acredite esta firmeza - normalmente una certificación emitida por parte del tribunal extranjero que ha dictado la resolución-, sino que ésta puede tanto derivarse de la propia resolución extranjera presentada como también de la ley aplicada por el tribunal de origen ${ }^{16}$. Precisa-

${ }^{12}$ En este sentido, puede consultarse el Auto de la Audiencia Provincial de A Coruña, de 28 de marzo de 2019, donde se rechaza el reconocimiento de una sentencia extranjera de divorcio, por no haber sido subsanado el defecto consistente en la falta de aportación de la sentencia debidamente legalizada o apostillada, pese a las múltiples ampliaciones de plazo concedidas, ECLI:ES:APC:2019:372A.

${ }^{13}$ La denegación del exequátur por la falta de esta exigencia documental, aunque referida al régimen aplicable contemplado en el Convenio entre España y Marruecos de 30 de mayo de 1997, fue el objeto del recurso de apelación resuelto por el auto de la Audiencia Provincial de Tarragona de 21 de enero de 2019, siendo éste último objeto de comentario por L.S. HEREDIA SANChez, "Procedencia de exequatur de sentencia extranjera de divorcio dictada en rebeldía: comentario al auto de la Audiencia Provincial de Tarragona, de 21 de enero de 2019", Cuadernos de Derecho Transnacional, n’.2, vol.11, 2019, pp.636-640.

${ }^{14}$ Lógicamente en las sentencias extranjeras de divorcio, como la del caso comentado, en donde únicamente se procede a disolver el vínculo matrimonial no hay nada que ejecutar.

${ }^{15}$ P. Martinez Llorente, “Comentario al artículo 54”, op.cit., p.467.

${ }^{16}$ En este último caso, se permite a las partes acreditar este requisito de la firmeza y, en su caso, fuerza ejecutiva, mediante la prueba de la vigencia y contenido del Derecho extranjero. Con ello la nueva regulación se muestra mucho más flexible que 
mente, valorando esta circunstancia en el caso concreto, la resolución comentada llega a la conclusión de que la firmeza consta y se extrae de la propia resolución extranjera "porque se trata de una sentencia de divorcio consensuado dictada en el marco de una postulación conjunta y de mutuo acuerdo entre los cónyuges. La sentencia de divorcio consigna expresamente la inexistencia de hechos controvertidos, siendo el pronunciamiento decretado consentido por ambas partes. No hay litigante vencido".

17. Por último, se requiere, siempre que sea preciso, las traducciones de los documentos presentados, de acuerdo con lo previsto en el artículo 144 de la LEC. La exigencia de traducción a lengua oficial española constituye un presupuesto para la eficacia probatoria de los documentos otorgados en el extranjero cuyo incumplimiento, tal y como establece el artículo 54.4 de la LCJIMC, puede dar lugar a la inadmisión de la demanda de exequátur. En el caso de la resolución comentada, aunque en el AAP de Barcelona se hace mención a esta necesaria presentación de las traducciones que, en principio, pueden ser privadas, sin perjuicio de la posible impugnación de las mismas por la parte demandada, lo que obligaría a una posterior traducción oficial, nada se precisa en el caso concreto, ya que la resolución argentina de divorcio viene ya redactada en idioma castellano.

18. En definitiva, con este AAP de Barcelona se corrige el error en el que incurrió el AJPI de Sant Boi de Llobregat al decretar el archivo de las actuaciones y, se procede acordar la admisión a trámite de la demanda de reconocimiento de la sentencia argentina de divorcio a sustanciar a través del procedimiento de exequátur. Con ello, en cierta medida, se mitigan los perjuicios derivados de la falta de un examen cuidadoso de los requisitos documentales precisos para la admisión de la demanda en primera instancia, lo que, por otra parte, ha pretendido ser reforzado por parte del artículo 54 en sus apartados 5, 6 y 7 de la LCJIMC, que prevén un primer examen por parte del letrado de la administración de justicia, un plazo de subsanación de cinco días para el supuesto de que los requisitos formales contemplados no se hubiesen cumplido y una decisión final del juez sobre la admisión o la inadmisión de la demanda de exequátur.

\footnotetext{
la anteriormente vigente. Así, por ejemplo, ello posibilita la presentación de informes o dictámenes elaborados por expertos en el Derecho extranjero, cuestión que ya había sido aceptada por parte del Tribunal Supremo, con anterioridad a la adopción de la LCJIMC, en su Auto de 18 de octubre de 2005 (JUR/2005/270947), FJ n ${ }^{\circ}$.4: "ha de tenerse por cumplido el requisito de la firmeza de la resolución, pues a los propios términos de la ejecutoria deben añadirse los de las declaraciones juradas que, en este punto, constituyen verdaderos dictámenes jurídicos sobre el derecho extranjero respecto del hecho de la firmeza y el carácter ejecutorio de la resolución".
} 